\title{
Moyamoya Disease, Basic Concepts of Diagnostics, and Treatment
}

\author{
Dilshod Mamadaliev, Alberto Feletti, \\ Tushit Mewada, Kei Yamashiro, Yasuhiro Yamada, \\ Tsukasa Kawase, and Yoko Kato
}

\subsection{Introduction}

Moyamoya disease (MMD) is a rare condition where the supraclinoid part of internal carotid artery is slowly and progressively becoming stenotic, and also it often involves both middle cere-

\section{Mamadaliev}

Department of Neurosurgery, Fujita Health University, Banbuntane Hotokukai Hospital, Nagoya city, Aichi, Japan

Republican Scientific Center of Neurosurgery, Tashkent, Uzbekistan

\section{A. Feletti}

Department of Neurosurgery, Fujita Health University, Banbuntane Hotokukai Hospital, Nagoya city, Aichi, Japan

Department of Neurosurgery, NOCSAE Modena Hospital, Modena, Italy

T. Mewada

Department of Neurosurgery, Fujita Health University, Banbuntane Hotokukai Hospital, Nagoya city, Aichi, Japan

G. B. Pant Institute of Post Graduate Medical Education and Research, New Delhi, India

K. Yamashiro $\cdot$ Y. Yamada $\cdot$ T. Kawase Department of Neurosurgery, Fujita Health University, Banbuntane Hotokukai Hospital, Nagoya city, Aichi, Japan

e-mail: kawasemi@hm8.aitai.ne.jp

\section{Y. Kato $(\bowtie)$}

Department of Neurosurgery, Fujita Health University, Toyoake, Aichi, Japan

e-mail: kyoko@fujita-hu.ac.jp bral arteries and anterior cerebral arteries (Fig. 26.1) [1]. The term moyamoya means a puff of smoke in Japanese, and it was first reported by Takeuchi and Shimizu in 1957 with Japanese language [2].

Its etiology still remains unknown. The moyamoya syndrome (MMS) has similar clinical and angiographic characteristics to MMD but might be associated with Down's syndrome, neurofibromatosis type 1 (NF-1), prior irradiation, and sickle-cell disease.

Although MMD was initially found in Asian patients, it has been increasingly reported in patients of all demographic groups; the exact incidence is not completely known [3].

Japanese studies report an annual incidence between 0.35 and $0.94 / 100,000$ persons per year and annual prevalence between 3.16 and 10.5 per 100,000 persons [4-6]. Female has twice the number compare with male $[4,5]$.

\subsection{Signs and Symptoms}

There is a classification that is supported by the Ministry of Health and Welfare of Japan, which classified the moyamoya diseases (MMD) based on its presentation in to four: ischemic, hemorrhagic, epileptic, and "other." In children, it is more common to see the ischemic type, while in the adult it is more common to see they present with hemorrhage. It is reported that 

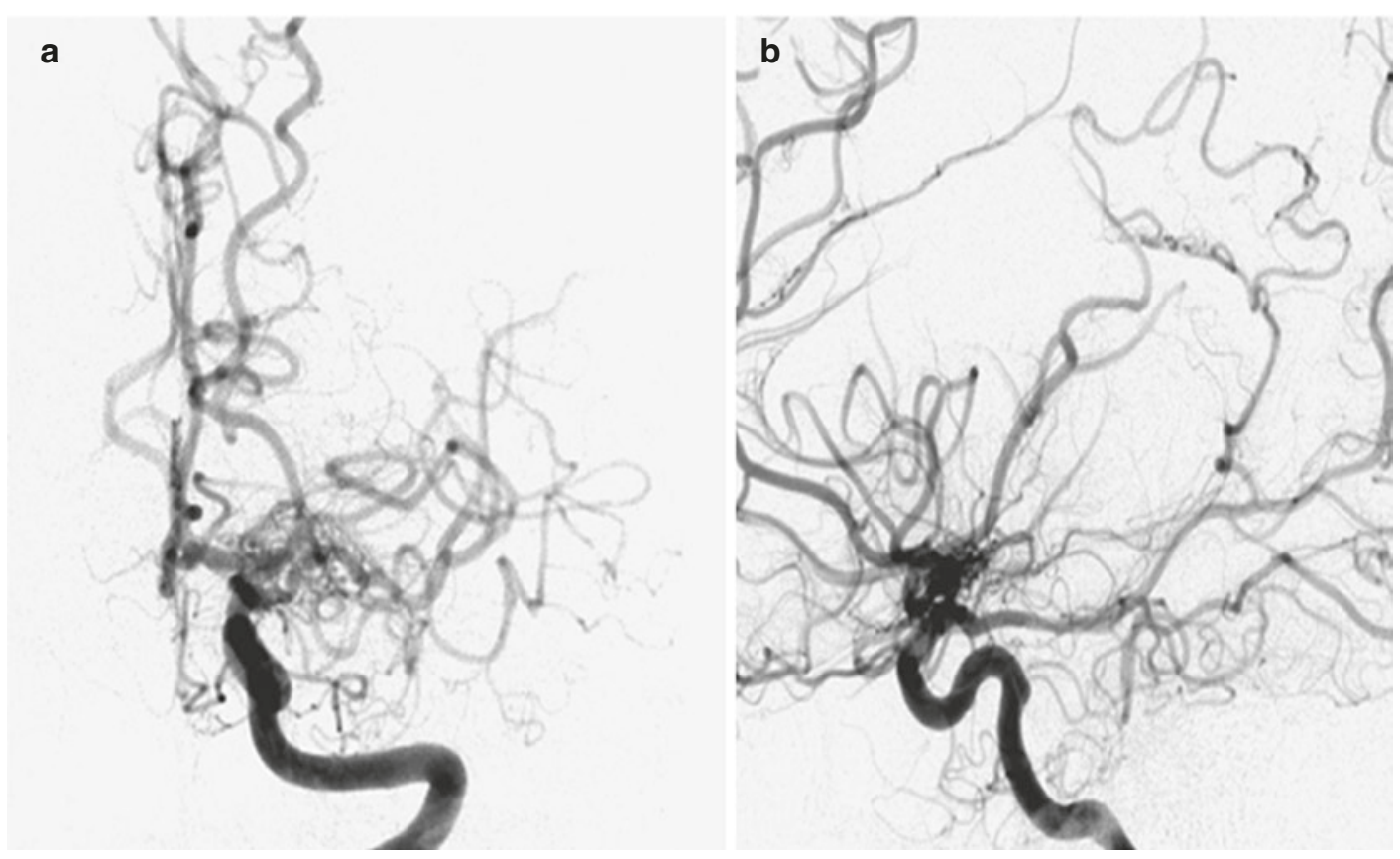

Fig. 26.1 Left ICA anterior-posterior and lateral injections demonstrating supraclinoid internal carotid artery stenosis and M1 occlusion with resultant moyamoya vessels

the hemorrhage presentation of MMD is approximately seven times higher in adult patients $(14.6 \%)$ if we compare with the pediatric group (2.1\%) [7]. Occasionally patients may also present with seizure, headaches, or behavioral changes. Some studies suggest a relation between headaches and hypoperfusion leading to cortical depression and associated migraine symptoms [8]. Others have suggested that dilation of dural and meningeal vessels irritate the pain fibers on the dura, resulting in a migraine-like headache that is refractive to medical therapies [9]. This headache initially persisted in $63 \%$ of patients even after cerebral revascularization, although it would often improve over time. Based on angiographic studies, the typical course of disease can be classified into six stages. The first description was written by Suzuki and Takaku (1969). They describe it in six stages. Stage I: on the early stage, it is started with the narrowing of carotid bifurcation at the neck and also the ICA bifurcation. Stage II: it is also called moyamoya initiation, which is characterized by dilated ACA, MCA, and further narrowing of ICA bifurcation. Stage III: this stage is called moyamoya intensifi- cation; the ICA bifurcation is much narrower, and ACA and MCA now become narrow as well. Stage $I V$ : this stage is called moyamoya minimization; the occlusive changes in ICA and tenuous ACA and MCA happened slowly. Stage V: This stage is called moyamoya reduction; the occlusion of ICA, ACA, and MCA becomes very severe. Stage VI: this stage is called moyamoya disappearance; the ICA is totally occluded, and the brain tries to get supplied from ECA.

On histopathologic examination, the internal carotid artery wall shows eccentric fibrocellular thickening of the intimal layer, and the smooth muscle cells proliferate too much. The ICA becomes tortuous, and frequently we will find the duplication of internal elastic lamina, and it does not show any inflammatory reaction or any atheromatous forming, resulting in artery stenosis/ occlusion [10]. The hypoxia induces supply from other parts to form collateral flow, and the arteries become tortuous and dilated (Fig. 26.2). These "moyamoya" vessels have many fibrin deposits at its wall, but the media layer is thin, the elastic lamina becomes fragmented, and so there 


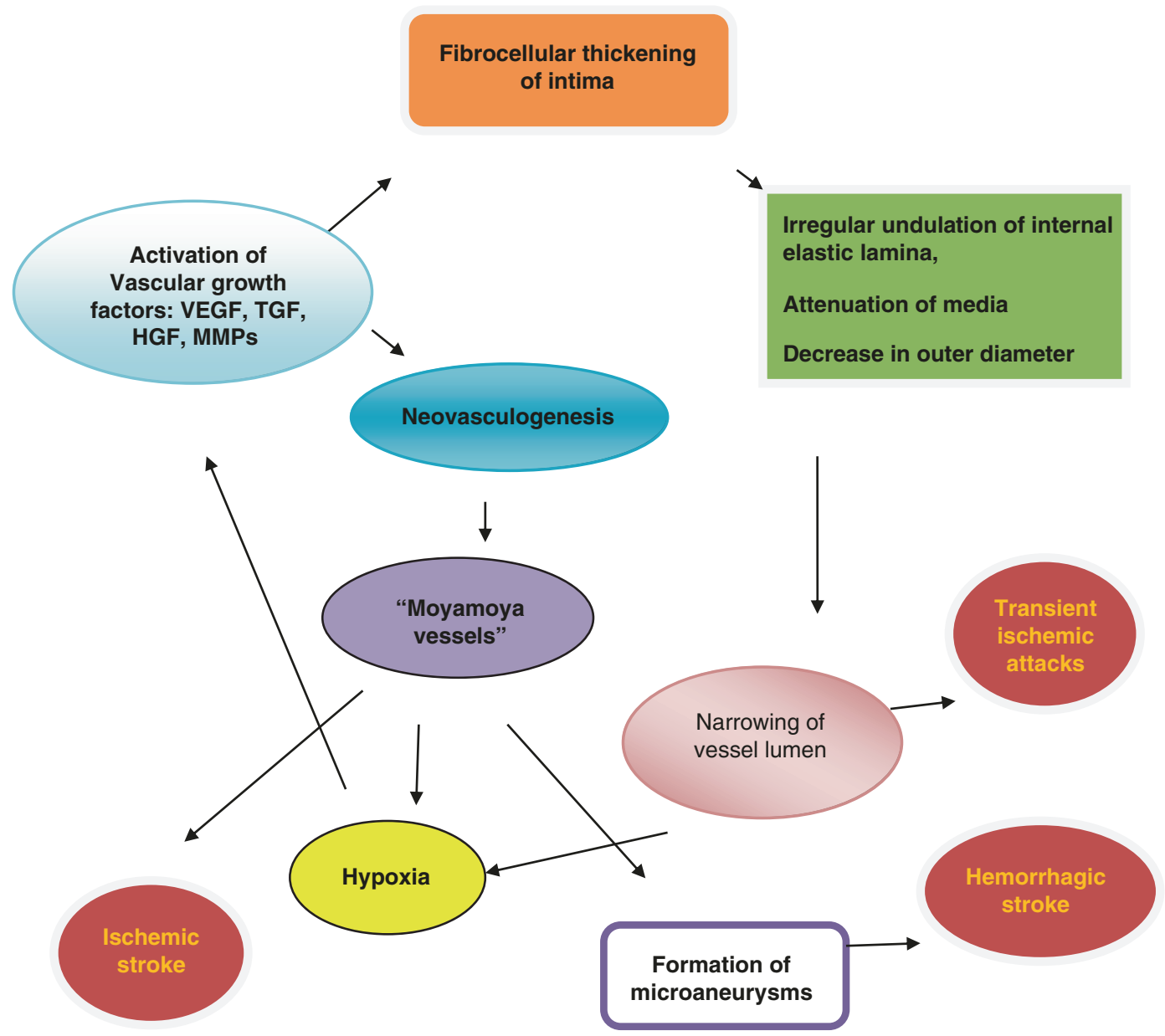

Fig. 26.2 Pathophysiology of moyamoya disease

is a high chance to have microaneurysm formation. It has been reported that the aneurysm formation might be associated with over-expression of several growth factors such as matrix metalloproteinases (MMPs), hypoxia-inducible factor 1 (HIF-1), vascular endothelial growth factor (VEGF), basic fibroblast growth factor (bFGF), transforming growth factor-beta (TGF-beta), and hepatocyte growth factor (HGF) [10]. It is also reported that autosomal dominant inheritance may play a role up to $15 \%$ of MMD patients, and some genome are widely studied from the family who carry the inheritance of MMD. The result shows some association with certain chromosomal regions including 3p24.2-p26 [11], 6q25 [12], 8q23 [13], 12p12 [13], and 17q25 [14].

\subsection{Natural History and Prognosis}

The disease inevitably progresses if left untreated. In a patient whose both side vessels are narrowing, the risk of stroke after 5 years is up to $82 \%$ [15]. The National Register of all asymptomatic moyamoya in Japan finds that $3.2 \%$ of them will get stroke even if they were treated medically [16]. On the other hand, the stroke or death rate prior, during, and after the surgical revascularization is something between $5.5 \%$ [7] and $17 \%$ [15] over 5 years, and this results in demonstrating that the surgery may offer a benefit to the patients. 


\subsection{Investigation}

The diagnoses of MMD or MMS should be considered in any patient, especially in a child or young adult who presents with neurologic deficits or symptoms secondary to cerebral ischemia or hemorrhage. Radiologic evaluation may include CT; MRI; cerebral angiography and cerebral perfusion studies, including CT perfusion, xenon-enhanced CT, or PET; MR perfusion; and SPECT or CT imaging without and with acetazolamide challenge. Cerebral angiography, CT angiography, and MR angiography imaging can reveal intracerebral vascular occlusion along with the resulting moyamoya vessels. $\mathrm{CT}$ and MRI can reveal hemorrhage or ischemic changes.

\subsection{Approach and Steps of Surgery}

Currently we still do not have a good randomized study that compares medical treatment versus surgical revascularization for patients with MMD, but in good hand surgeon, they claim that the stroke rate is significantly reduced in surgically treated group. As discussed in the previous section, the natural history of these patients is to progress, and the risk of recurrent stroke is extremely high with medical management only. The main medical treatment option for these patients is antiplatelet therapy. All patients are on aspirin pre- and postoperatively. Full anticoagulation drugs such as warfarin are rarely used.

One of the obvious indications to recommend surgery for moyamoya patients is multiple episodes of TIA (transient ischemic attack), RIND (reversible ischemic neurological deficit), or even stroke. The purpose of the surgery is to prevent the next episode by reestablishing adequate blood supply to the affected cortex. There are two ways to reestablish the blood supply to the brain. Adult and older children may have direct bypass surgery to revascularize the brain vessels, and the children under 10 years old may have indirect bypass sur- gery because their vessels are very tiny and tend to fail if we do direct bypass.

\subsubsection{Bypass Surgery of the Brain}

Bypass surgery of the brain is a revascularization either between extracranial vessel and intracranial vessel or between intracranial vessel and another intracranial vessel by using graft (saphenous vein, radial artery, etc.) The purpose of the bypass is to reestablish another route or reroute blood flow from the narrowed, blocked, or damaged vessel and supply the distal part of the artery. It is very common to just use the natural provided artery such as from the STA (superficial temporal artery) and directly bypass it to the MCA (middle cerebral artery). Just after the bypass, it may show an instant upgrading blood flow to the distal part of the narrowed artery. Certain people may have a very tiny superficial temporal artery, and we might think the size is not big enough to provide long-term support, and then saphenous vein or radial artery may be harvested to make an anastomosis from the extracranial carotid artery to the distal part of the narrowed vessel. The surgeon will look at the angiogram or nowadays the $\mathrm{CT}$ angiogram to decide and plan the surgery.

\subsubsection{EDAS (Encephaloduroarterio- synangiosis)}

EDAS (encephaloduroarteriosynangiosis) surgery is considered as an indirect way of establishing new vascularization in the brain. It is using the superficial temporal artery that was dissected from the surrounding soft tissue and is placed in direct contact with the cortical surface. The artery should not be kinking at its entry point to the cortical surface and at its distal part where it is going back to supply the scalp. Some fine suture should be placed to hold the artery on the cortical surface. The bone flap is repositioned and secured with miniplate and screw, but the entry and exit of the STA have to make sure there is no pressure at all. Over a period, we expect some new 
vessel forming to supply the brain. It is important to remember that the STA is always kept intact, so it will give branches to the brain.

\subsubsection{EMS}

\section{(Encephalo-Myo-Synangiosis)}

EMS (encephalo-myo-synangiosis) surgery is also considered as indirect way of establishing new vascularization by using temporalis muscle as the source of the angiogenesis to the brain. The muscle is gently dissected, and through the opening of the bone at the temporal base, the muscle is laid on top of the cortical surface. It is very important to have a meticulous bleeding control to avoid postoperative blood accumulation, but be careful not to coagulate too much of the temporal muscle because we don't want to make the muscle de-vascularized. Closing the dura with the muscle underneath it is sometimes challenging, but surgeons need to do the closing meticulously. The muscle is a very good source of blood supply to the brain, and they may spread angiogenesis all over the brain.

\subsubsection{EDAMS (Encephalo-Duro- Arterio-Myo-Synangiosis)}

EDAMS (encephalo-duro-arterio-myo-synangiosis) surgery is a combination EDAS and EMS at the same time.

\subsubsection{Omental Transposition Surgery}

Omental transposition surgery is also another option for indirect way to establish revascularization in the brain by using omentum from the abdomen. The omentum is a special fat tissue that surrounds abdominal organs, and it spreads very fast to any part of the abdomen that needs support. It has rich blood supply. When it is placed on the surface of the cerebral cortex, it is expected that it will grow and spread very fast to supply the brain and improve the blood supply.

\subsubsection{Multiple Burr Holes}

Another option of indirect revascularization of the cortex is by doing multiple burr holes all over the skull and expecting that the scalp vessel will go through the burr hole and provide vascular supply to the cortex.

Patients with MMD although have occlusion of the internal carotid arteries, but usually the external carotid artery is patent. Surgical effort uses the external carotid artery to supply blood to ischemic cortex by either creating a direct or indirect revascularization bypass. A direct bypass usually require adequate superficial temporal artery (STA) at the ipsilateral side and then anastomosed directly to a distal M4 segment of middle cerebral artery (MCA). The indirect revascularization is performed by using a vascularized tissue that is also supplied by external carotid artery, including the dura, temporalis muscle, the STA itself, and even pericranium. The vascularized tissue is laid on the cortical surface and expect the new blood vessel will be formed and ingrowth toward the cortex. There is no good randomized study that compares the direct and indirect revascularization in terms of their safety and efficacy. The literature review also failed to reveal a difference in surgical morbidity or stroke rate (perioperative or long term) between the direct and indirect methods. Many institutions will perform direct revascularization for adults and indirect revascularization for children because of the smaller and more friable caliber of the vessels in children. However, there are many groups that perform indirect bypass for adult patients as well [15, 16]. According to Gordon Li et al., they recommend to perform direct bypass on all patients whenever possible, unless the STA or MCA is too small or friable. Direct revascularization surgery may provide an immediate increase in cortical blood flow and treat the ischemic cortex immediately. In the case with bilateral MMD, it is obvious to treat the symptomatic side first. If they are both asymptomatic, it is better to treat and revascularize the non-dominant hemisphere first because of higher incidence of transient neurologic symptoms following the surgery on dominant cortex. 
The second surgery for the contralateral side is generally 1-4 weeks after the first surgery, unless there were significant complications after the first procedure.

\subsubsection{Direct Revascularization}

Prior to the surgery, it is important to assess the size of frontal and parietal branch of STA by using CT angiography or conventional angiography. The larger diameter branch is chosen as the donor vessel. The patient is positioned supine and the head is hold with head holder. The head is elevated above the heart and turned to the side, bringing the frontotemporal region uppermost in the field. Stimulating and recording electrodes are placed for monitoring of bilateral somatosensory evoked potentials (SSEP) and bilateral EEG. Cooling to approximately $33{ }^{\circ} \mathrm{C}$ is accomplished by either using cooling blanket and bladder irrigation or by putting IV catheter (InnerCool Therapies Philips, Andover, MA) into the inferior vena cava. In general, the InnerCool catheter is placed in the larger patients who are more difficult to cool and rewarm via surface techniques.

The course of the superficial temporal artery is plotted by using a Doppler probe. When parietal branch of STA is chosen as donor, the skin incision is planned over the artery along its course (Fig. 26.3). If the frontal branch is the donor, the inferior portion of the incision follows the STA, but as the artery tracks anterior to the hairline, the incision stays posterior behind the hairline for cosmetic purposes. After shaving the hair over the planned incision, prepare and drape the surgical area. The operating microscope is positioned and used for careful harvesting of the STA. A length of approximately $7 \mathrm{~cm}$ of STA together with a generous cuff of soft tissue to protect it is needed for the bypass (Fig. 26.4). Then the underlying temporalis fascia muscle is split and dissected from the frontotemporal bone. Burr holes are placed, the craniotomy bone flap is removed at frontotemporal area, and the dura is cut in a cruciate manner (Fig. 26.5).

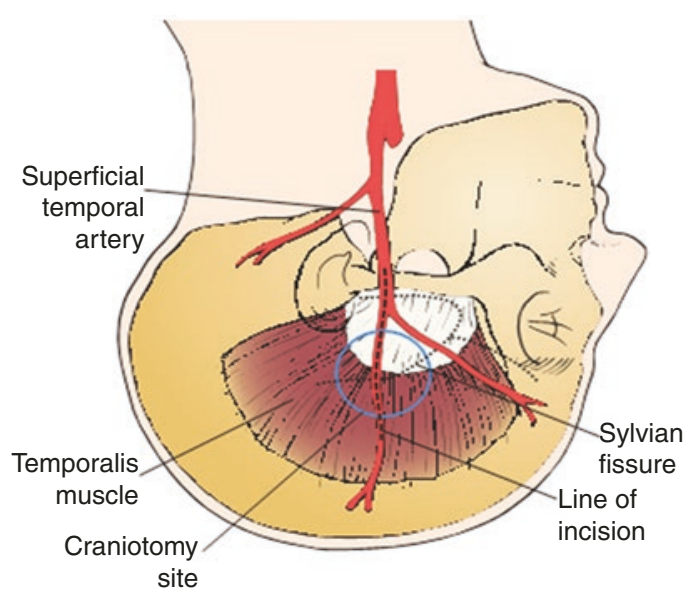

Fig. 26.3 The course of superficial temporal artery is mapped out using Doppler ultrasound, and skin incision is planned over the artery along its course

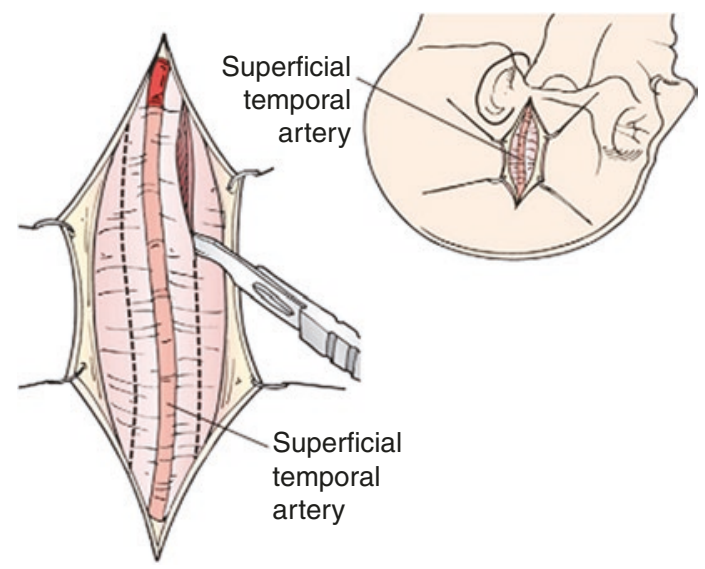

Fig. 26.4 Approximately $7 \mathrm{~cm}$ of STA with a generous cuff of soft tissue to protect is needed for the bypass. The artery and cuff are carefully dissected out using a combination of sharp dissection and cautery

Identification of a sufficiently large M4 segment of MCA $(0.6 \mathrm{~mm})$ emerging from Sylvian fissure is paramount for this procedure. The arachnoid overlying this cortical branch of the MCA is then microscopically opened. A $7 \mathrm{~mm}$ segment of M4 artery without branches is preferably chosen as the recipient, but any tiny branches arising from this segment can be coagulated and divided if necessary (Fig. 26.6). A jeweler-type bipolar is used. High-visibility background is placed under the M4 segment. Papaverine is intermittently instilled over the vessels to prevent 


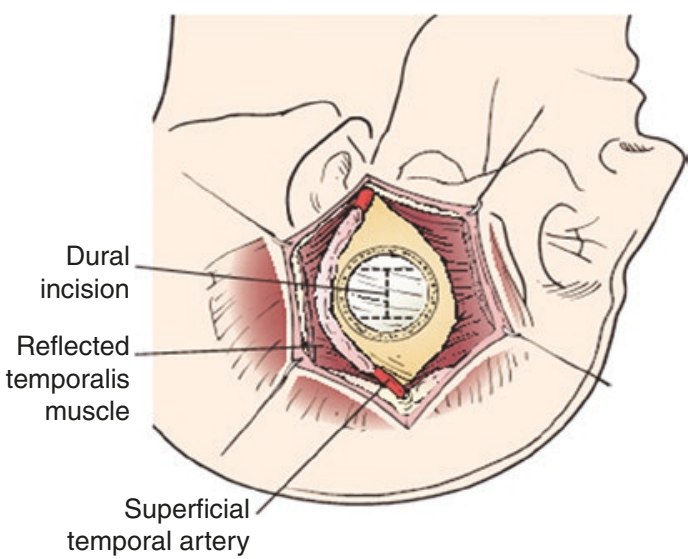

Fig. 26.5 The underlying temporalis fascia muscle is split and dissected from the frontotemporal bone. Burr holes are placed, the craniotomy bone flap is removed over the frontotemporal region, and the dura is opened in a cruciate manner

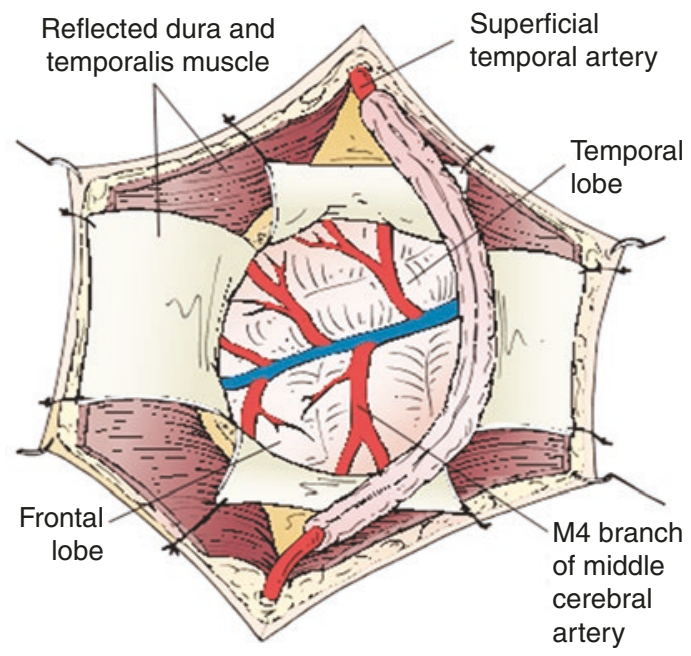

Fig. 26.6 Identification of a sufficiently large M4 branch of the MCA $(0.6 \mathrm{~mm})$ emerging from the Sylvian fissure is paramount for this procedure

spasm. Then the STA is temporarily occluded proximally and sectioned distally. The distal stump of the STA in the scalp is coagulated, and the STA is truncated to the proper length for anastomosis and fish mouthed. Temporary release of the proximal clip is performed to ensure excellent flow.

The artery is temporarily occluded again proximally and flushed with heparinized saline. Intravenous thiopental is given to induce burst suppression in the EEG. The mean arterial pressure is raised, and then the M4 MCA branch is temporarily occluded with Sugita aneurysm clips. An arteriotomy is made in the M4 branch, removing an elliptical portion of the superior wall, and irrigated with heparinized saline. Anastomosis between an end of STA and the side of the MCA segment is done with 10-0 interrupted suture (Fig. 26.7). The Sugita clips are then removed, restoring flow. Flow in all vessels is confirmed with the intraoperative Doppler. An intraoperative indocyanine green (ICG) angiogram is performed by injecting $2 \mathrm{ml}$ of ICG dye intravenously and visualizing the graft with the near-infrared camera on the microscope.

If the anastomosis is done correctly, the ICG demonstrates wide patency of the graft with no stenosis and good filling of the pial vasculature. It is our practice to then lay the STA with its soft tissue cuff on the cortical surface to induce an indirect bypass as well as a direct one (Figs. 26.8 and 26.9). The dura is closed with 4-0 suture followed by synthetic dura, leaving an opening for the graft to enter without compromise. The bone is replaced using a plating system, also leaving an opening for the graft to enter unimpeded. The temporalis muscle and scalp are closed in several layers in the usual fashion. During the entire procedure, care is taken to keep the mean arterial pressure high normal for that patient and about ten points higher during the occlusion of the cortical MCA branch for adequate perfusion. Patients are maintained normocapnia during the entire operation to prevent vasoconstriction and ischemia from hyperventilation.

\subsubsection{Indirect Revascularization}

If the patient has a very small STA $(<0.6 \mathrm{~mm})$ or the vessel is too fragile for suture, then we perform an indirect revascularization (encephaloduroarteriosynangiosis (EDAS) or encephaloduromyosynangiosis (EDMS)). We have abandoned options such as free omental flap transplantation or pedicle omental flap transposition. In a similar fashion to the direct bypass procedure, patients are positioned and prepped, the 
a

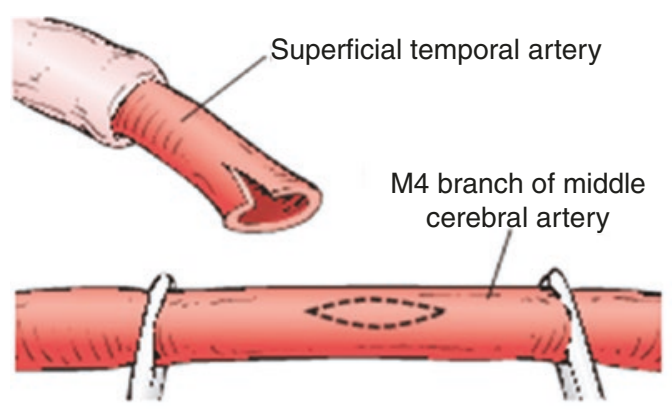

C

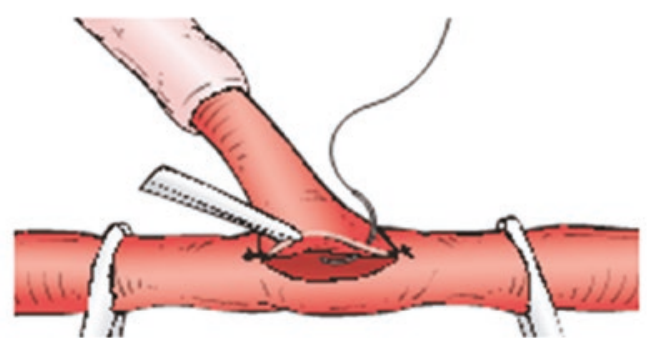

Fig. 26.7 (a) The superficial temporal artery is truncated to the proper length for anastomosis and fish mouthed. (b) An arteriotomy is made in the M4 branch, removing an

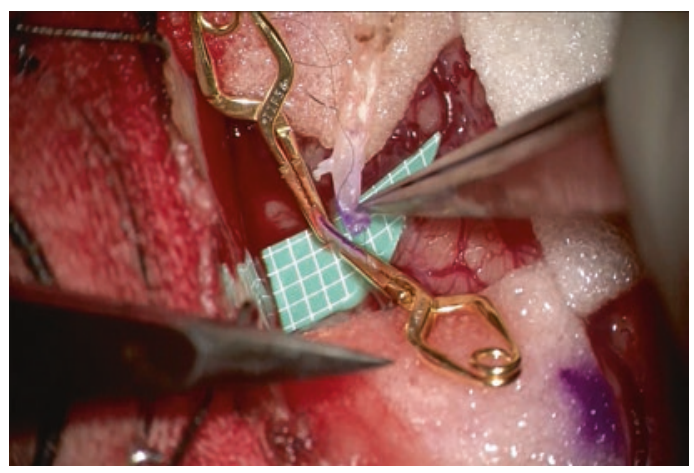

Fig. 26.8 Intraoperative photo of STA-MCA bypass procedure b

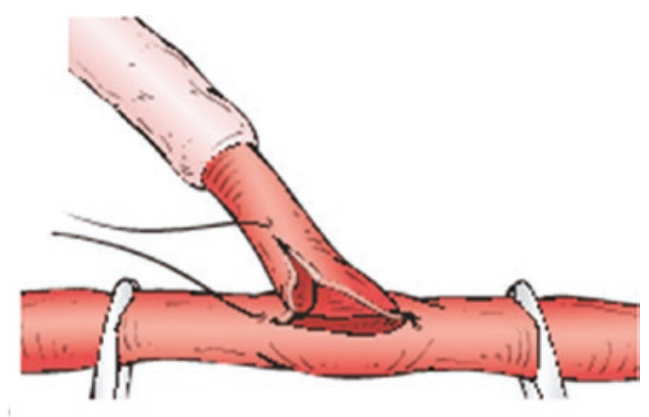

d

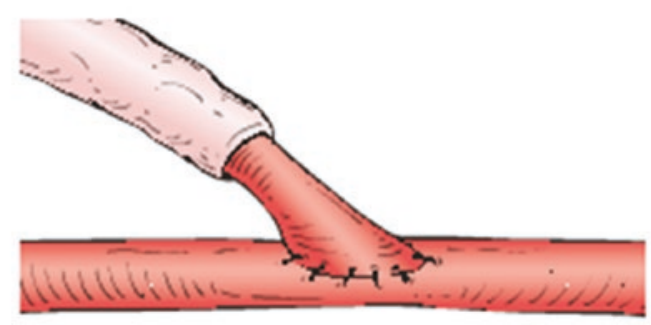

elliptical portion of the superior wall. (c and d) An end-toside anastomosis between the STA branch and the MCA branch is performed with 10-0 interrupted suture

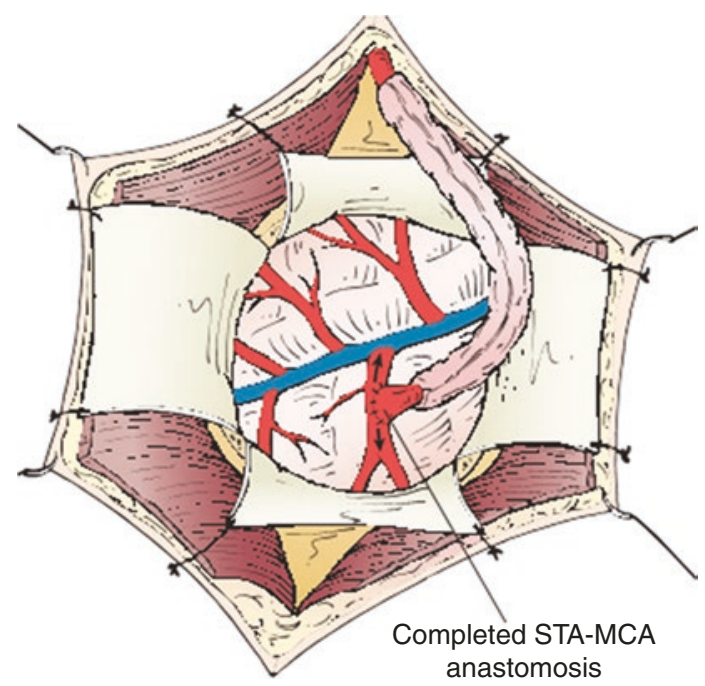

Fig. 26.9 Completed bypass showing the STA with its soft tissue cuff on the cortical surface to induce both an indirect and direct bypass 
STA and generous soft tissue cuff are harvested, and the craniotomy is performed. The arachnoid overlying the deep sulci and fissures is microscopically opened, and the STA with the cuff of soft tissue is laid directly on the pial surface of the cortex. The closure should be meticulously done to avoid postoperative bleeding.

\subsection{Expert Opinion}

Although there are risks to direct and indirect revascularization procedures for MMD, according to data by several authors, they suggest that surgery is superior to medical management of these patients.

\subsection{Things to Be Observed and Postoperative Care/ Follow-Up}

Patients are generally discharged after three to four hospital nights (the first night is spent in the intensive care unit). They are then evaluated clin- ically 1 week after each surgery and subsequently at 6 months, then 3 years, and finally at 10 years. It is important to perform MRI, SPECT, or Xenon CT and cerebral angiography at 6 months, 3 years, and 10 years.

In addition to internal carotid and vertebral injections, injections of external carotid artery are important to evaluate the bypass patency and extent of revascularization (Fig. 26.10). Patients who presented with TIA (transient ischemic attack) or stroke trended toward an increased risk of postoperative ischemic symptoms. Similarly, patients who presented with hemorrhage trended toward an increased risk of postoperative hemorrhage. Be aware that the MMD patient has a higher risk of postoperative stroke and death compared to a non-MMD patient. Sex, ethnicity, unilateral versus bilateral, age, and type of revascularization procedure do not affect outcome [7]. In another study, the 5-year risk of stroke or death was decreased from $65 \%$ in medically treated patients to $17 \%$ in surgically treated patients [15]. One study did fail to show a benefit of surgical treatment, but the authors reported an unusually high initial surgical morbidity and mortality [16].
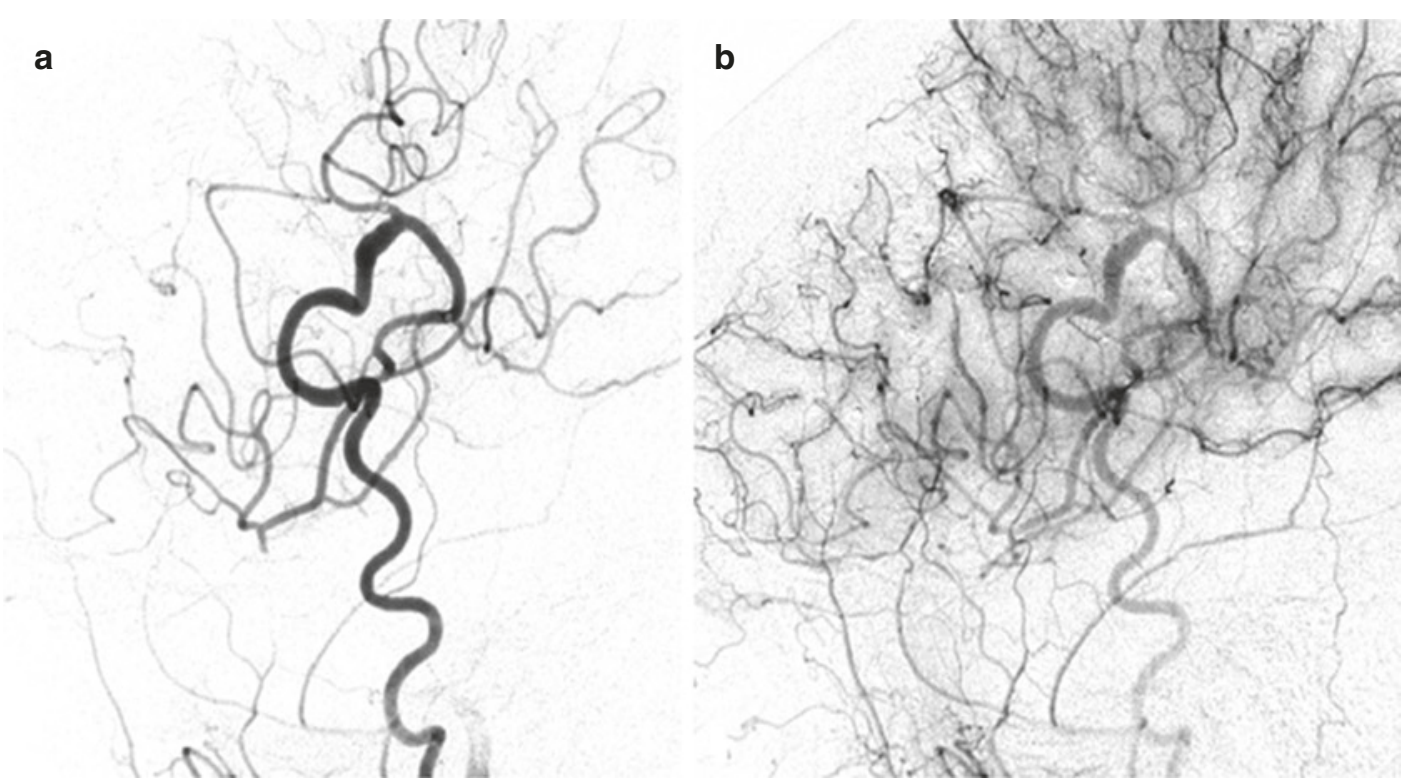

Fig. 26.10 (a and b) Left external carotid artery lateral injection demonstrating a patent superficial temporal artery to middle cerebral artery bypass supplying two thirds of the MCA territory 


\section{References}

1. Fukui M. Guidelines for the diagnosis and treatment of spontaneous occlusion of the Circle of Willis ("Moyamoya" disease). Research Committee on Spontaneous Occlusion of the Circle of Willis (Moyamoya disease) of the Ministry of Health and Welfare, Japan. Clin Neurol Neurosurg. 1997;99(Suppl 2):S238-40.

2. Takeuchi K, Shimizu K. Hypoplasia of the bilateral internal carotid arteries. Brain Nerve. 1957;9: 37-43.

3. Hoffman HJ. Moyamoya disease and syndrome. Clin Neurol Neurosurg. 1997;99(Suppl 2):S39-44.

4. Baba T, Houkin K, Kuroda S. Novel epidemiological features of moya-moya disease. J Neurol Neurosurg Psychiatry. 2008;79:900-4.

5. Wakai K, Tamakoshi A, Ikezaki K, et al. Epidemiological features of moyamoya disease in Japan: findings from a nationwide survey. Clin Neurol Neurosurg. 1997;99(Suppl 2):S1-5.

6. Kuriyama S, Kusaka Y, Fujimura M, et al. Prevalence and clinic-epidemiological features of moyamoya disease in Japan: findings from a nationwide epidemiological survey. Stroke. 2008;39:42-7.

7. Guzman R, Lee M, Achrol A, et al. Clinical outcome after 450 revascularization procedures for moyamoya disease. J Neurosurg. 2009;111:927-35.

8. Olesen J, Friberg L, Olsen TS, et al. Ischaemiainduced (symptomatic) migraine attacks may be more frequent than migraine-induced ischemic insults. Brain. 1993;116(Pt 1):187-202.

9. Seol HJ, Wang KC, Kim SK, et al. Headache in pediatric moyamoya disease: review of 204 consecutive cases. J Neurosurg. 2005;103:439-42.

10. Achrol AS, Guzman R, Lee M, et al. Pathophysiology and genetic factors in moyamoya disease. Neurosurg Focus. 2009;26:E4.

11. Ikeda H, Sasaki T, Yoshimoto T, et al. Mapping of a familial moya-moya disease gene to chromosome 3p24.2-p26. Am J Hum Genet. 1999;64:533-7.

12. Inoue TK, Ikezaki K, Sasazuki T, et al. Linkage analysis of moyamoya disease on chromosome 6. J Child Neurol. 2000;15:179-82.

13. Sakurai K, Horiuchi Y, Ikeda H, et al. A novel susceptibility locus for moyamoya disease on chromosome 8q23. J Hum Genet. 2004;49:278-81.

14. Mineharu Y, Liu W, Inoue K, et al. Autosomal dominant moyamoya disease maps to chromosome 17q25.3. Neurology. 2008;70:2357-63.

15. Hallemeier CL, Rich KM, Grubb RL Jr, et al. Clinical features and out-come in North American adults with moyamoya phenomenon. Stroke. 2006;37:1490-6.

16. Kuroda S, Hashimoto $\mathrm{N}$, Yoshimoto $\mathrm{T}$, et al. Radiological findings, clinical course, and outcome in asymptomatic moyamoya disease: results of multicenter survey in Japan. Stroke. 2007;38: $1430-5$.

Open Access This chapter is licensed under the terms of the Creative Commons Attribution 4.0 International License (http://creativecommons.org/licenses/by/4.0/), which permits use, sharing, adaptation, distribution and reproduction in any medium or format, as long as you give appropriate credit to the original author(s) and the source, provide a link to the Creative Commons license and indicate if changes were made.

The images or other third party material in this chapter are included in the chapter's Creative Commons license, unless indicated otherwise in a credit line to the material. If material is not included in the chapter's Creative Commons license and your intended use is not permitted by statutory regulation or exceeds the permitted use, you will need to obtain permission directly from the copyright holder. 